\title{
Partial flap loss in transgender phalloplasty using the anterolateral thigh or forearm - a systematic literature review
}

\author{
Isabel Cylinder ${ }^{1}$, Aaron Heston ${ }^{1}$, Breanna Jedrzejewski², Zbigniew Sikora', Blair Peters ${ }^{3}$, Jens Urs Berli² \\ 'School of Medicine, Oregon Health \& Science University, Portland, OR 97239, USA. \\ 2Division of Plastic Surgery, Oregon Health \& Science University, Portland, OR 97239, USA. \\ ${ }^{3}$ Division of Plastic and Reconstructive Surgery, Washington University School of Medicine in St. Louis, MO 63110, USA.
}

Correspondence to: Dr. Jens Urs Berli, Division of Plastic Surgery, Oregon Health \& Science University, 3303 SW Bond Avenue, Portland, OR 97239, United States. E-mail: berli@ohsu.edu

How to cite this article: Cylinder I, Heston A, Jedrzejewski B, Sikora Z, Peters B, Berli JU. Partial flap loss in transgender phalloplasty using the anterolateral thigh or forearm - a systematic literature review. Plast Aesthet Res 2020;7:58.

http://dx.doi.org/10.20517/2347-9264.2020.85

Received: 20 Apr 2020 First Decision: 18 May 2020 Revised: 23 May 2020 Accepted: 19 Jun 2020 Published: 24 Oct 2020

Academic Editor: Marlon E. Buncamper, Stan J. Monstrey Copy Editor: Cai-Hong Wang Production Editor: Jing Yu

\begin{abstract}
Aim: In this study, we systematically review the current literature regarding partial flap loss (PFL) for the two most commonly performed types of phalloplasty, the radial forearm and the anterolateral thigh flaps. The primary purpose is to synthesize the available information to clarify anatomic location, etiology, extent of flap loss, and management thereof. Second, we utilize this information to inform strategies to mitigate the risk of PFL.
\end{abstract}

Methods: A systematic review of all abstracts published on phalloplasty on PubMed was performed. Abstracts were reviewed by two senior authors who included all studies discussing flap-related outcomes after radial forearm free flap (RFFF) phalloplasty or anterolateral thigh flap (ALT) phalloplasty for the treatment of gender dysphoria. Primary variables collected include: flap type, PFL rate, anatomic location, extent of and management of PFL.

Results: A total of 17 papers that reported on RFFF and/or ALT phalloplasty were included. A total of 780 RFFF and 182 ALT phalloplasties were identified. The PFL rate was $4.5 \%$ and $7.1 \%$ respectively. Only $4 / 17$ papers commented on the anatomic location of PFL; none commented on the exact extent of PFL and only 4/17 commented on the management of PFL.

\footnotetext{
(6) (1)

(C) The Author(s) 2020. Open Access This article is licensed under a Creative Commons Attribution 4.0 International License (https://creativecommons.org/licenses/by/4.0/), which permits unrestricted use, sharing, adaptation, distribution and reproduction in any medium or format, for any purpose, even commercially, as long as you give appropriate credit to the original author(s) and the source, provide a link to the Creative Commons license, and indicate if changes were made.
}

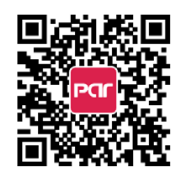


Conclusion: The current literature suggests a higher rate of PFL in the ALT cohort (7.1\% vs. 4.5\%). The information available on PFL lacks detail as to the anatomic location, extent, and management of this complication. Future studies should strive to report on the above variables and include pertinent patient demographics and flap characteristics that may affect the rates and management of PFL. This information will assist in optimizing outcomes.

Keywords: Phalloplasty, transgender, flap loss, complications, gender dysphoria

\section{INTRODUCTION}

Phalloplasty as a surgical treatment for gender dysphoria was pioneered by Sir Harold Gillies and has been performed since $1946^{[1]}$. Early techniques pre-dated the current era of mainstream microsurgery and relied on adjacent tissue transfer in the form of loco-regional pedicled flaps, typically tubed abdominal flaps ${ }^{[2]}$. As microsurgical techniques evolved and perforator flap dissection became clinically routine, tissue from more distant locations became available for the construction of a neophallus ${ }^{[3-5]}$. With better-suited skin and soft tissue quality and the ability for improved innervation, outcomes have improved significantly. This has ushered in the current era of phalloplasty for the treatment of gender dysphoria, where the focus is on achieving the best possible phallus reconstruction while minimizing overall morbidity. While many donor sites have been explored and are still currently utilized, the radial forearm and the anterolateral thigh are the most commonly used in phalloplasty surgery for gender dysphoria ${ }^{[6-9]}$ [Figures 1 and 2]. Current techniques and surgical staging vary widely between institutions and individual surgeons, thus making it difficult to draw conclusions regarding the frequency and extent of complications. Furthermore, this makes it challenging to reach a consensus on best practice regarding the management of these complications and the sequelae of phalloplasty for the treatment of gender dysphoria.

The overall complication rate in phalloplasty for the treatment of gender dysphoria is extraordinarily high, with urologic complications being the most frequent ${ }^{[10,11]}$. While there are an abundance of publications regarding the management of urologic sequelae, the current body of literature suffers from a paucity of articles that report on flap-related complications in detail. Additionally, the majority of evidence available is in the form of case reports and retrospective case series. Currently, randomized controlled trials and cohort studies are exceedingly rare in this field of practice. Larger systematic reviews have been performed but due to the heterogenous nature of surgical techniques, staging, and extent of reporting in the current body of literature, they often lack granularity ${ }^{[12]}$.

One complication that suffers from the above shortcomings in the literature is that of partial flap loss (PFL). This is often only mentioned in a list of complications or as an overall percentage. With the size of flaps used for phalloplasty often extending beyond their traditional dimensions, it is no surprise that reported rates are relatively high. On anecdotal literature review, we noticed that beyond incidence, there is very little discussion regarding anatomic location, etiology, or extent of PFL, or the management of PFL when it occurs. We therefore felt it was timely to systematically analyze the current literature for the two most commonly performed types of phalloplasty: radial forearm free flap (RFFF) and anterolateral thigh flap (ALT) to determine an overall PFL rate following these procedures. Next, we sought to synthesize the currently available information regarding the anatomic location, extent, etiology, and management of PFL. We then utilized this information to inform a discussion on the current literature and outline future directions regarding risk mitigation and the management of PFL following phalloplasty for the treatment of gender dysphoria. 


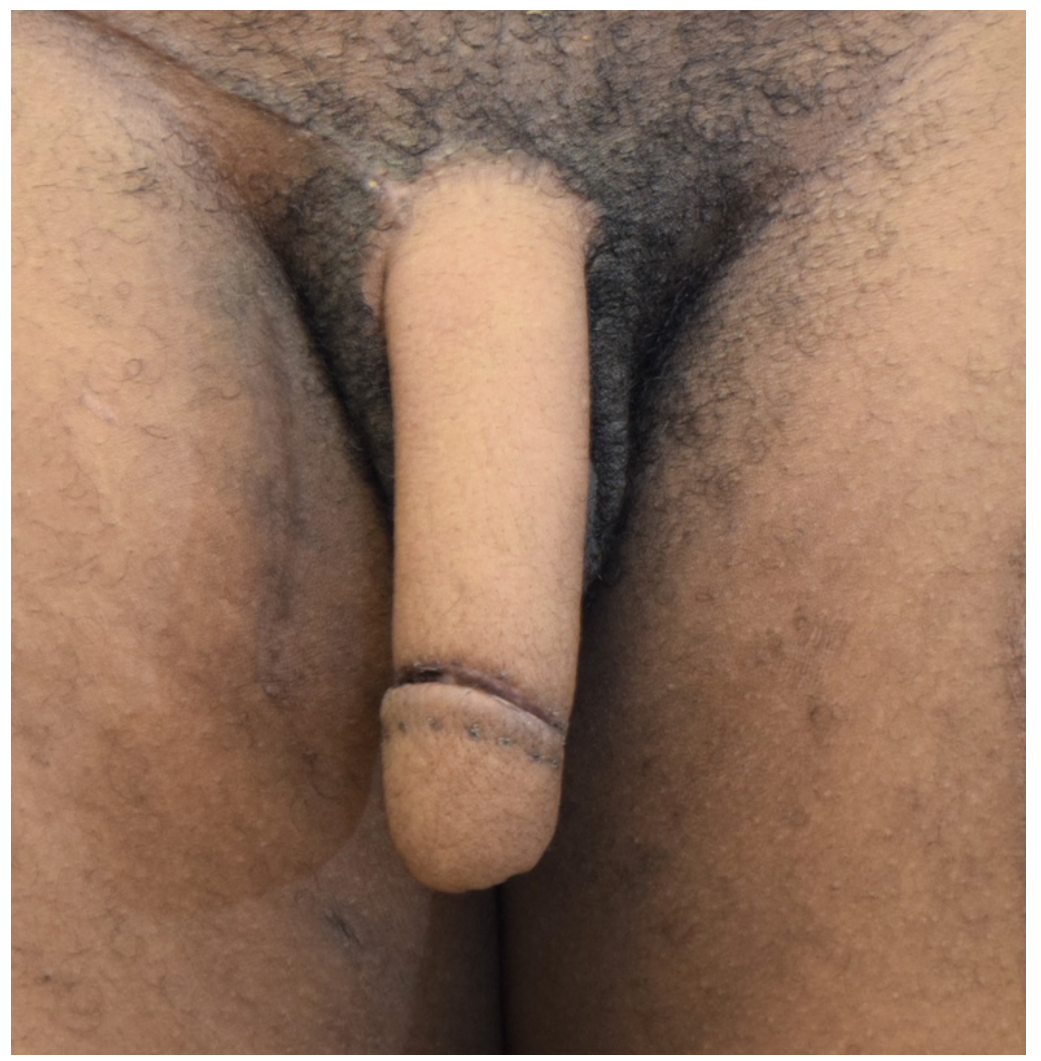

Figure 1. Results after radial forearm free flap phalloplasty

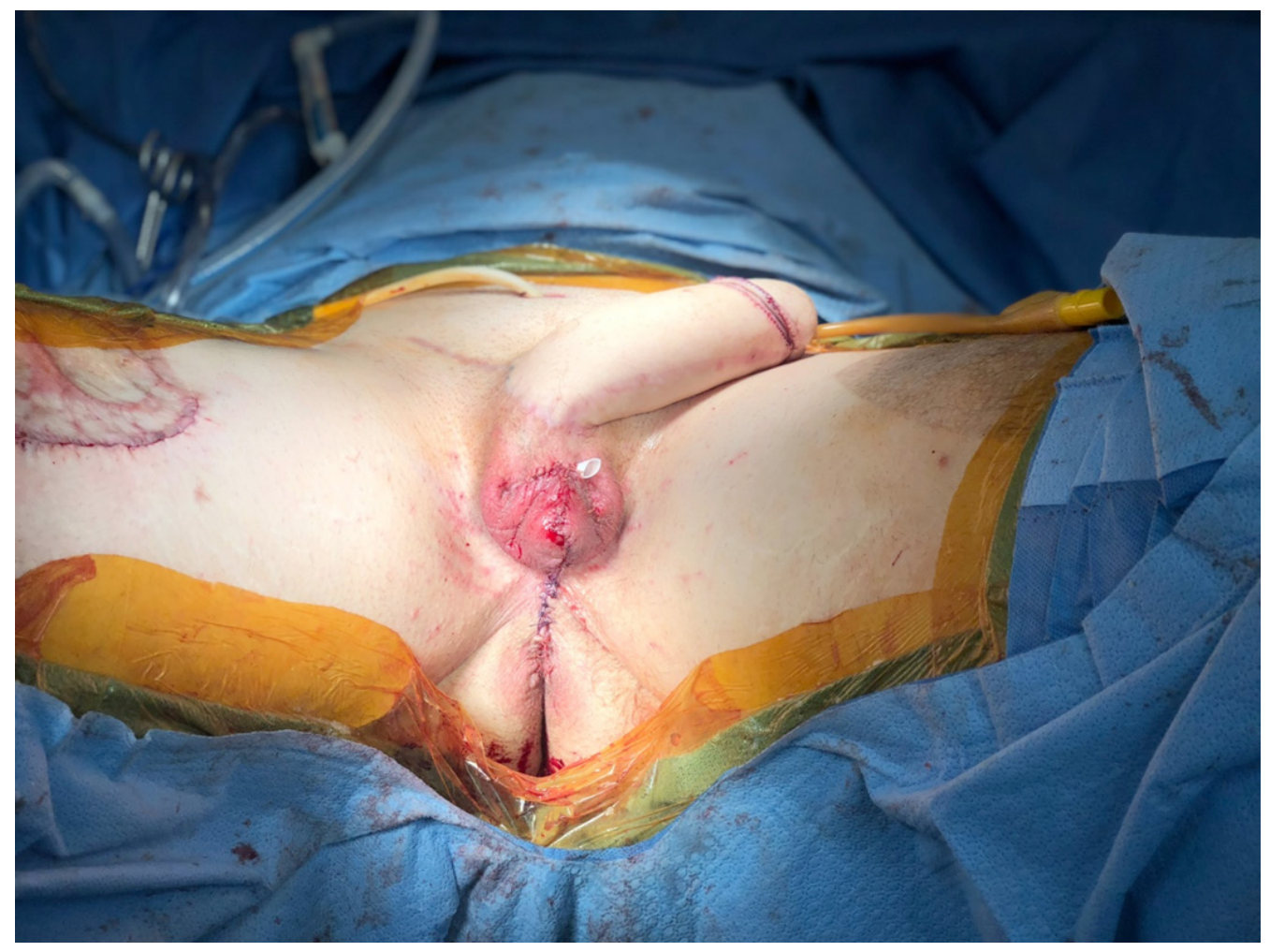

Figure 2. Result after antero-lateral thigh flap phalloplasty ${ }^{[3,34]}$ 


\section{METHODS}

A systematic literature review of all articles published on the topic of phalloplasty up to and including March 2020 was performed using the PubMed database. Our search was designed to capture all published studies that fulfill our inclusion criteria of presenting primary data on the flap-related outcomes of RFFF or ALT phalloplasty as a gender-affirming surgical procedure. RFFF and ALT were defined as the main flap used for shaft creation. Exclusion criteria included: case reports, review articles; phalloplasty performed for reasons other than gender-affirming care; phalloplasty performed using techniques that did not include either RFFF or ALT, or did not specify the technique used for reconstruction; and articles that did not include flap outcomes in the dataset (i.e., urologic outcomes following phalloplasty, prosthesis outcomes following phalloplasty). Articles published in any journal in both the English and German languages were considered. Data were collected on patient demographics, flap design, partial flap loss, total flap loss, timing of glansplasty, location of flap loss and management thereof.

The search term used was "phalloplasty". All titles were screened by the senior author and a second author with experience in the field of transgender surgery. Articles that met the exclusion criteria were dismissed. All remaining articles were then screened for abstract content by four reviewers. Any additional article that met the exclusion criteria was subsequently removed. The remaining articles were then formally reviewed and all articles that met the inclusion criteria were selected for this review. By including the date range for each article, we tried to make sure there was no double reporting from the same institution and patients were not accidentally counted twice. If a later publication included the time period of an earlier one, then the earlier publication was excluded. See Figure 3 for an overview of the systematic review and Tables 1-3 for results.

\section{RESULTS}

A total of 421 publications were identified in the initial literature review. Of these, 56 abstracts were relevant to phalloplasty for the treatment of gender dysphoria and thus were screened systematically. 29 papers were determined to meet the inclusion criteria and were fully reviewed. Following full review, only 17 papers had RFFF and/or ALT phalloplasties in the specified cohort and clearly indicated rates of partial flap loss [Figure 3]. All articles were retrospective case series. A total of 1,199 phalloplasties, consisting of 836 RFFF and 210 ALT flaps, were identified in these 17 papers. The collective PFL rate for all flap types included in these studies was $6.1 \%$ [Table 1$]^{[13-29]}$.

Subgroup analysis was performed to determine the rate of PFL in RFFF and ALT flaps respectively. On subgroup analysis, articles that did not specify flap type when reporting partial PFL rates were excluded $\mathrm{d}^{[13,21,26]}$. A total of 780 RFFF were identified from 11 articles, with a PFL rate of $4.5 \%$ [Table 2] $]^{[14,15,17-19,22-25,27,29]}$. A total of 182 ALT flaps were identified from four articles, with a PFL rate of $7.1 \%$ [Table 3$]^{[14,16,20,28]}$.

Only 4/17 papers commented on the anatomic location of PFL. All four of these papers pertained to RFFF phalloplasty (RFFFP) and reported necrosis of the distal tip. No papers commented on the exact extent of PFL when an anatomic location was mentioned, though Fang et al. ${ }^{[18]}$ reported $10 \%$ reduction in length of the neophallus as an outcome for one patient following PFL.

Only 4/17 papers commented on the management of PFL when it occurred. Two of these papers pertained to RFFF and two pertained to ALT. Management of PFL following RFFFP was reported as healing by secondary intention or debridement and VAC therapy followed by skin grafting by Song et al ${ }^{[27]}$, or repeat RFFFP by Baumeister et al. ${ }^{[15]}$. Management of PFL following ALT flap phalloplasty was reported as splitthickness skin grafting by van der Sluis et al. ${ }^{[28]}$, or debridement and skin grafting by D’Arpa et al. ${ }^{[16]}$. No outcome articles included any photographs. 


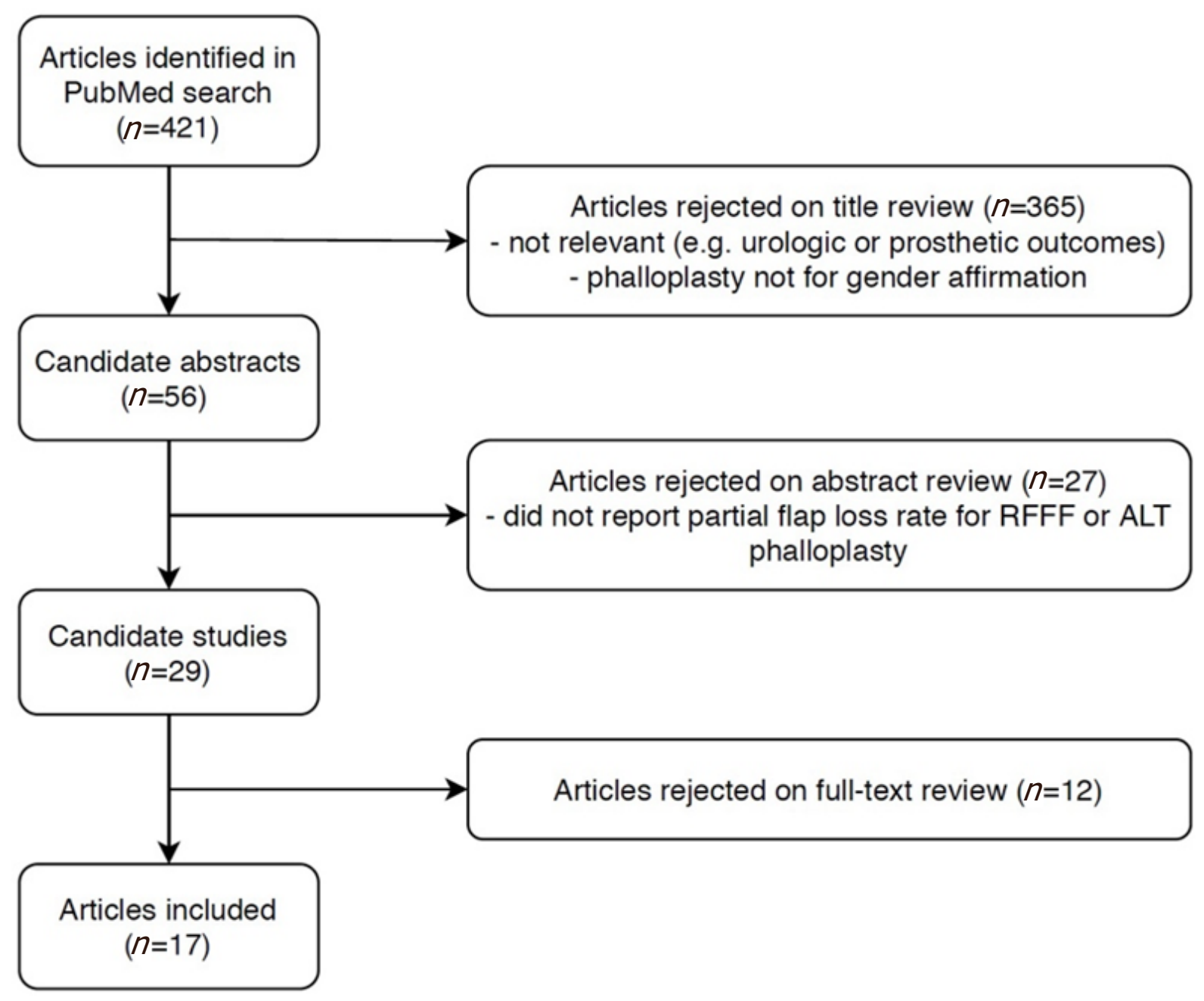

Figure 3. Flowchart demonstrating the process of identifying, including and rejecting studies to be utilized in this systematic review

\section{DISCUSSION}

Traditionally, phalloplasty as a surgical treatment for gender dysphoria has only been offered by a small group of surgeons at a few select centers around the world. These groups have been instrumental in developing the techniques that we are currently using today. The body of literature that has amassed over the past four decades focuses mainly on the various potential donor sites and grouped outcomes of a variety of technical modifications, with a persisting majority consensus that the radial forearm presents the current donor site of choice. Typically, the anterolateral thigh is the most common alternative to the radial forearm. Amassed experience with these two donor sites for phalloplasty in the transgender population has allowed phalloplasty to go from a relatively experimental procedure to a somewhat standardized set of procedures to achieve masculine-appearing and functional external genitalia. However, in the grand scheme of reconstructive surgery, phalloplasty is still a relatively rare procedure. Due to the various donor sites utilized across the world and the breadth of technical variations on flap design, harvest, shaping, and different variations regarding surgical stages, the literature lacks further insight into the various aspects of phalloplasty. A large portion of the literature focuses on reporting the successful use of various donor sites, with the take-home message that phalloplasty can be performed with success. As a surgical community treating patients with gender dysphoria, we are just beginning to progress past this more superficial perspective. It is imperative that we move beyond just broad reporting of complication rates and begin to include data on the severity of specific complications along with the impact and management 


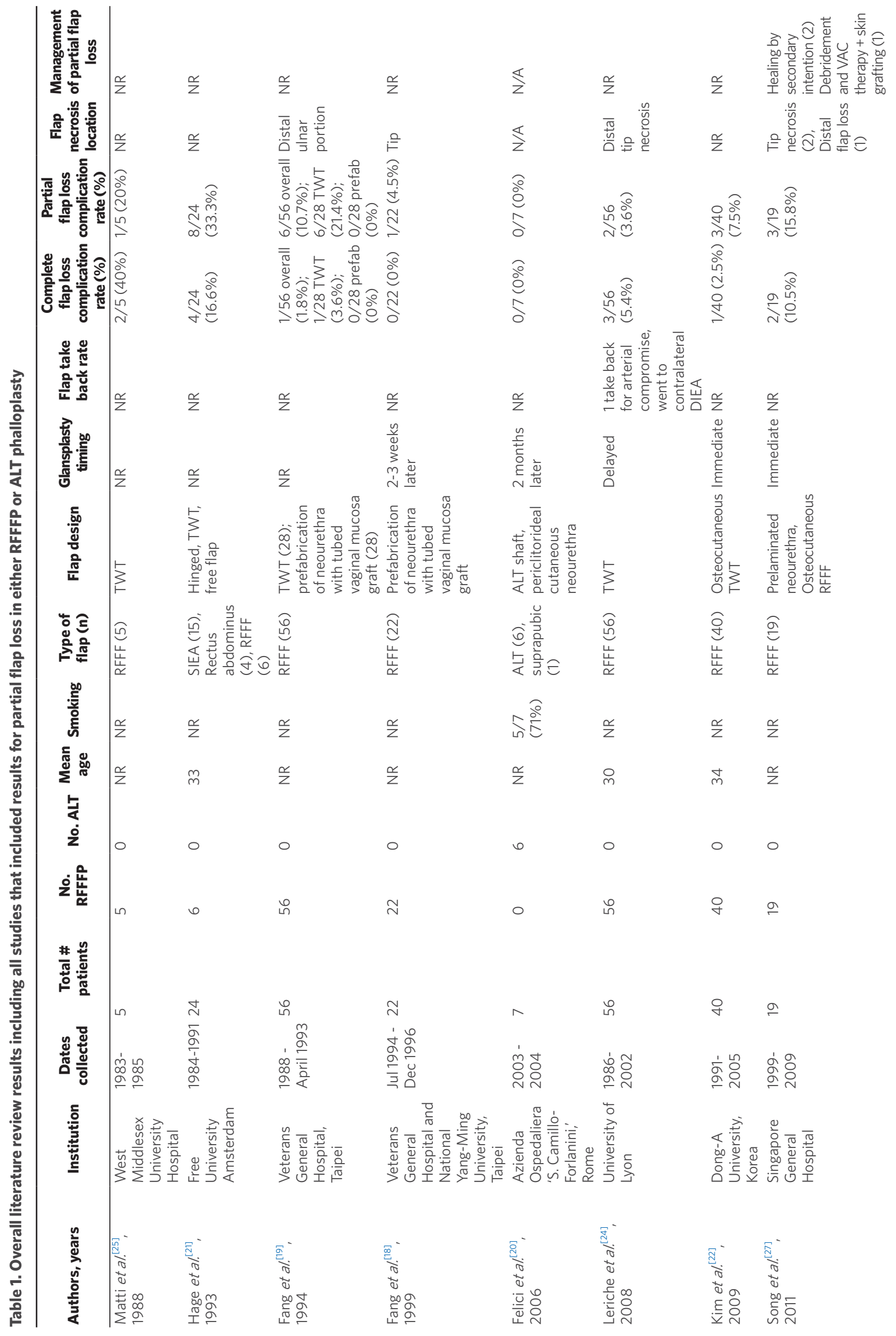




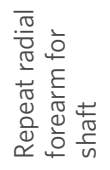

$\frac{\sqrt[4]{\pi}}{\frac{\pi}{n}} \Xi$

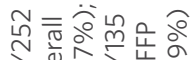

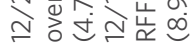

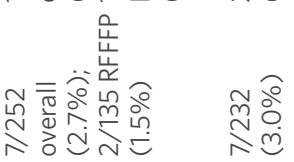

ָָฐิ

$\frac{o}{z}$

సָे

\section{号}

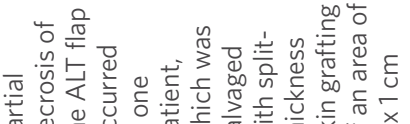

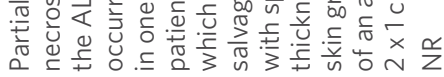

$\frac{\varangle}{z} \frac{\alpha}{z}$

은

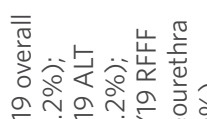

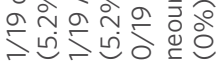

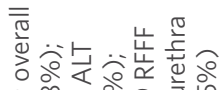

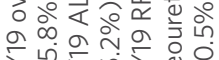

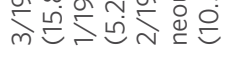

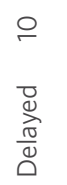

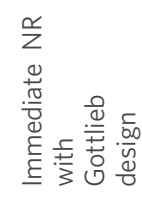

$\stackrel{\propto}{z}$

兴

产

$\stackrel{\frac{1}{z}}{z}$

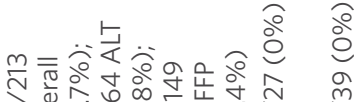

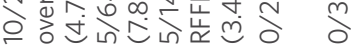

兴

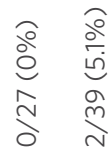

$\stackrel{\propto}{z}$

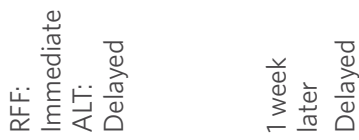

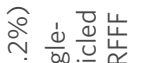

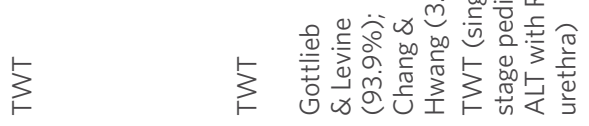

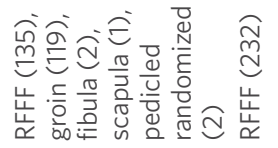

ฟิ

文

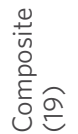

ํํㅇ

ㅇ

○

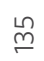

กี

ṁo

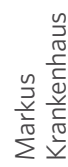

ลิ

$\underset{\frac{0}{2}}{2}$

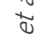

离

搴 $\bar{N}$
응

ָี

$\stackrel{\infty}{m}$

○

g

○

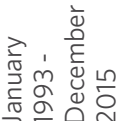

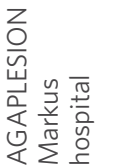



in

g

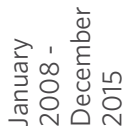

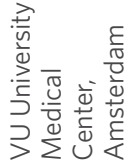

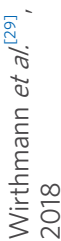

$\lessgtr$

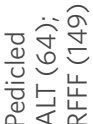

吉上享星品宫

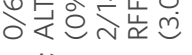

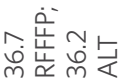

క్ర

g

$\stackrel{m}{\sim}$

ํㅡㅁ

촌른

品

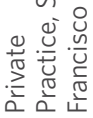

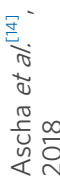

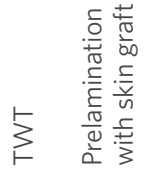

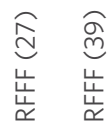

«

₹ $\stackrel{\infty}{\dot{j}}$

$\circ 0$

స

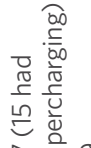

출

₹

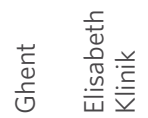

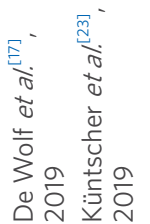




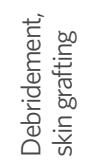

$\stackrel{\Upsilon}{z}$

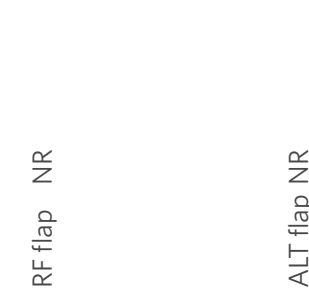

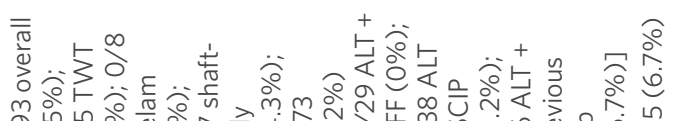

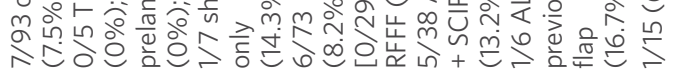

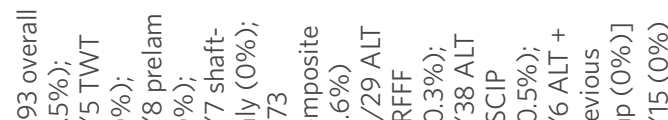

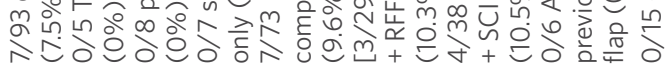

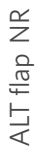

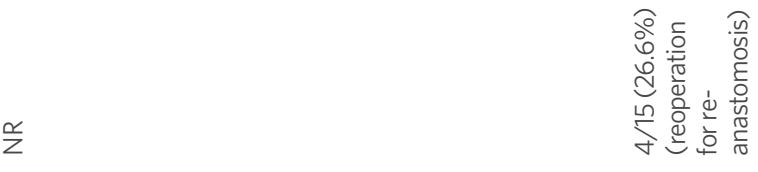

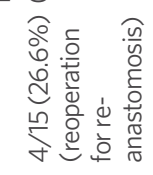

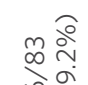

bิ

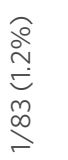

离

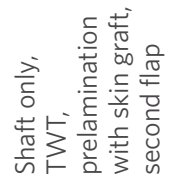

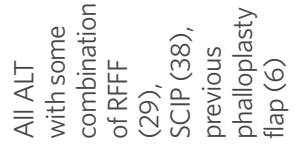

ํํㄴ

兄孚

兴

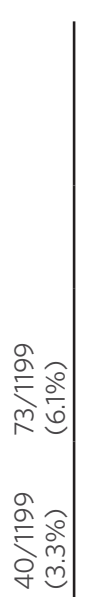

$\frac{8}{8}$

产

(n)

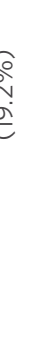


Table 2. Extracted data for RFFFP partial flap loss used for shaft reconstruction

\begin{tabular}{|c|c|c|c|c|c|}
\hline \multicolumn{6}{|c|}{ Radial forearm free flap phalloplasty } \\
\hline Flap design & \# patients & Total flap loss (\%) & Partial flap loss (\%) & Necrosis location & Management \\
\hline TWT & 672 & $16 / 672(2.4 \%)$ & $31 / 672(4.6 \%)$ & - & - \\
\hline Matti et al. ${ }^{[25]}, 1988$ & 5 & $2 / 5(40 \%)$ & $1 / 5(20 \%)$ & NR & NR \\
\hline Fang et al. ${ }^{[19]}, 1994$ & 28 & $1 / 28(3.6 \%)$ & $6 / 28(21.4 \%)$ & Distal ulnar portion & NR \\
\hline $\begin{array}{l}\text { Leriche et } a l^{[24]}, \\
2008\end{array}$ & 56 & $3 / 56(5.4 \%)$ & $2 / 56(3.6 \%)$ & Distal tip necrosis & NR \\
\hline Kim et al. ${ }^{[22]}, 2009$ & 40 & $1 / 40(2.5 \%)$ & $3 / 40(7.5 \%)$ & NR & NR \\
\hline $\begin{array}{l}\text { Baumeister et al. }{ }^{[15]}, \\
2011\end{array}$ & 135 & $2 / 135(1.5 \%)$ & $12 / 135(8.9 \%)$ & Shaft & Repeat RFFFP \\
\hline $\begin{array}{l}\text { Wirthmann et al. } \\
2018\end{array}$ & 232 & $7 / 232(3.0 \%)$ & $2 / 232(0.9 \%)$ & NR & NR \\
\hline Ascha et al. ${ }^{[14]}, 2018$ & 149 & NR & $5 / 149(3.4 \%)$ & NR & NR \\
\hline $\begin{array}{l}\text { De Wolf et al. }{ }^{[17]}, \\
2019\end{array}$ & 27 & $0 / 27(0 \%)$ & $0 / 27(0 \%)$ & $N / A$ & $N / A$ \\
\hline Prefab urethra & 108 & 4/108 (3.7\%) & 4/108 (3.7\%) & - & - \\
\hline Fang et al. ${ }^{[19]}, 1994$ & 28 & $0 / 28(0 \%)$ & $0 / 28(0 \%)$ & $\mathrm{N} / \mathrm{A}$ & $\mathrm{N} / \mathrm{A}$ \\
\hline Fang et al. ${ }^{[18]}, 1999$ & 22 & $0 / 22(0 \%)$ & $1 / 22(4.5 \%)$ & Tip & NR \\
\hline Song et $a l_{.[27]}, 2011$ & 19 & $2 / 19(10.5 \%)$ & $3 / 19(15.8 \%)$ & $\begin{array}{l}\text { Tip necrosis (2); Distal flap } \\
\text { loss (1) }\end{array}$ & $\begin{array}{l}\text { Healing by secondary } \\
\text { intention ( } 2 \text { ) } \\
\text { Debridement and VAC } \\
\text { therapy + skin grafting (1) }\end{array}$ \\
\hline $\begin{array}{l}\text { Küntscher et al. }{ }^{[23]}, \\
2019\end{array}$ & 39 & $2 / 39(5.1 \%)$ & $0 / 39(0 \%)$ & $\mathrm{N} / \mathrm{A}$ & NR \\
\hline Total & 780 & $20 / 780(2.6 \%)$ & $35 / 789(4.5 \%)$ & - & - \\
\hline
\end{tabular}

RFFFP: radial forearm free flap phalloplasty; TWT: tube within a tube design; NR: Not reported; N/A: not applicable; VAC: vacuumassisted closure of wound

Table 3. Extracted data for ALT partial flap loss used for shaft reconstruction

\begin{tabular}{|c|c|c|c|c|c|c|}
\hline \multicolumn{7}{|c|}{ Anterolateral thigh flap phalloplasty } \\
\hline Flap design & \# patients & Pedicled vs. free & Total flap loss (\%) & Partial flap loss (\%) & Necrosis location & Management \\
\hline TWT & 69 & & $0 / 69(0 \%)$ & $5 / 69(7.2 \%)$ & - & - \\
\hline Ascha et al. ${ }^{[14]}, 2018$ & 64 & Pedicled & NR & $5 / 64(7.8 \%)$ & NR & NR \\
\hline D'Arpa et al. ${ }^{[16]}, 2019$ & 5 & Pedicled ${ }^{\star}$ & $0 / 5(0 \%)$ & $0 / 5(0 \%)$ & $\mathrm{N} / \mathrm{A}$ & NA \\
\hline Prefab urethra & 8 & & $0 / 8(0 \%)$ & $0 / 8(0 \%)$ & - & - \\
\hline D'Arpa et al. ${ }^{[16]}, 2019$ & 8 & Pedicled* & $0 / 8(0 \%)$ & $0 / 8(0 \%)$ & N/A & N/A \\
\hline Composite & 98 & & $10 / 98(10.2 \%)$ & $7 / 98(7.1 \%)$ & - & - \\
\hline Felici et al. ${ }^{[20]}, 2006$ & 6 & Free & $0 / 6(0 \%)$ & $0 / 6(0 \%)$ & N/A & N/A \\
\hline $\begin{array}{l}\text { van der Sluis et al. }{ }^{[28]} \text {, } \\
2017\end{array}$ & 19 & Pedicled & $\begin{array}{l}\text { 1/19 complete ALT } \\
\text { loss ( } 5.2 \%) \\
2 / 19 \text { complete RFFF } \\
\text { neourethral losses } \\
(10.5 \%)\end{array}$ & $1 / 19(5.2 \%)$ & NR & $\begin{array}{l}\text { Partial necrosis } \\
\text { of the ALT flap } \\
\text { occurred } \\
\text { in one patient, which } \\
\text { was salvaged with } \\
\text { split-thickness skin } \\
\text { grafting of an area of } \\
2 \mathrm{~cm} \times 1 \mathrm{~cm}\end{array}$ \\
\hline D'Arpa et al. ${ }^{[16]}, 2019$ & 73 & Pedicled ${ }^{\star}$ & $\begin{array}{l}7 / 73(9.6 \%) \\
{[3 / 29 \text { ALT + RFFF }} \\
(10.3 \%) ; 4 / 38 \text { ALT } \\
\text { + SCIP }(10.5 \%) ; \\
\text { 0/6 ALT + previous } \\
\text { flap (0\%)] }\end{array}$ & $\begin{array}{l}6 / 73(13.2 \%) \\
\text { [0/29 ALT + RFFF } \\
\text { (0\%); 5/38 ALT + } \\
\text { SCIP (13.2\%); } 1 / 6 \\
\text { ALT + previous flap } \\
(16.7 \%)]\end{array}$ & NR & $\begin{array}{l}\text { Debridement, skin } \\
\text { grafting }\end{array}$ \\
\hline Shaft-only & 7 & & $0 / 7(0 \%)$ & $1 / 7(14.3 \%)$ & - & - \\
\hline D'Arpa et al. ${ }^{[16]}, 2019$ & 7 & Pedicled* & $0 / 7(0 \%)$ & $1 / 7(14.3 \%)$ & NR & NR \\
\hline TOTAL & 182 & & $10 / 182(5.5 \%)$ & $13 / 182(7.1 \%)$ & - & - \\
\hline
\end{tabular}

TWT: tube within a tube design; NR: not reported; N/A: not applicable; ALT: anterolateral thigh flap; RFFF: radial free flap; SCIP: superficial circumflex iliac perforator 
of said complications. This has, in part, been done more successfully regarding the urologic complications following phalloplasty, but less so for flap-related complications. Total flap loss remains the most feared complication of phalloplasty. This particular complication is self-explanatory, "total" meaning the entire flap is non-viable. Additionally, "partial flap loss" has been routinely reported, but few studies mention how "partial" is defined. "Partial" can refer to a variety of flap involvement, spanning from a simple issue such as minor marginal flap necrosis to a major complication with large volumes of tissue loss leading to multiple revision surgeries and impaired long-term aesthetic and functional outcomes. This was demonstrated in the literature review with the term "partial flap loss" being used to describe both small areas of distal necrosis and cases that mandated an additional free flap. This reinforces the vague definition of "partial flap loss" that plagues the current literature. Beyond the issue of a lack of consistent and meaningful reporting, there is very little offered in the literature regarding the mitigation of PFL in phalloplasty procedures. Based on this, we felt that a systematic review was a good first step to assess what we currently know about PFL, specifically regarding location, extent, and management of PFL.

\section{Etiology and reduction of risk for development of partial flap loss}

While the current data is lacking for some of the following statements, we would like to suggest the following etiologies for PFL in RFFFP and ALT phalloplasty, respectively.

(A) For all flaps:

(1) Patient selection: It is well established that certain medical co-morbidities or drug/substance abuse can affect PFL rate (e.g., peripheral vascular disease and diabetes, smoking and cocaine, etc. ${ }^{[30]}$.

(2) Flap design that includes dimensions beyond the capacity of perforators included in the flap and therefore relies on random pattern circulation for areas of tissue that are too far removed from the included perfasomes.

(3) Technical error by inadvertently excluding or injuring perforating vessels during flap design and harvest.

(B) Specific to RFFFP:

(1) Failure to include the critical and sparse proximal radial artery perforators during flap harvest and only relying only on distal perforators [Figure 4].

(2) Positioning of the flap design that fails to place the radial artery as close to the center of the flap as possible.

(C) Specific to ALT phalloplasty:

(1) Failure to include or the absence of distal perforators.

(2) Inability to position the flap distal enough on the thigh allowing adequate pedicle length for transposition while still including an adequate number of perforators to support the volume of tissue harvested.

(3) Excessive intra-operative thinning or excessive size of flap harvested with tissue take beyond the perfusion capacity of the perfasomes.

(4) Not delaying the flap when there is concern regarding adequacy of perfusion. Delay will assist in flap maturation and improve perfusion characteristics.

\section{A1 patient selection}

The mean age of patients included in the systematic review was 34.6 years. Given the increasing accumulation of medical co-morbidities with age, this speaks to the fact that overall, this is a predominantly healthy patient population. Unfortunately, none of the articles mentioned the age of patients suffering from PFL. Smoking is a well-established risk factor for PFL and other associated wound healing issues at both the recipient and donor sites ${ }^{[30]}$. We were surprised to find that some centers offer phalloplasty to active 


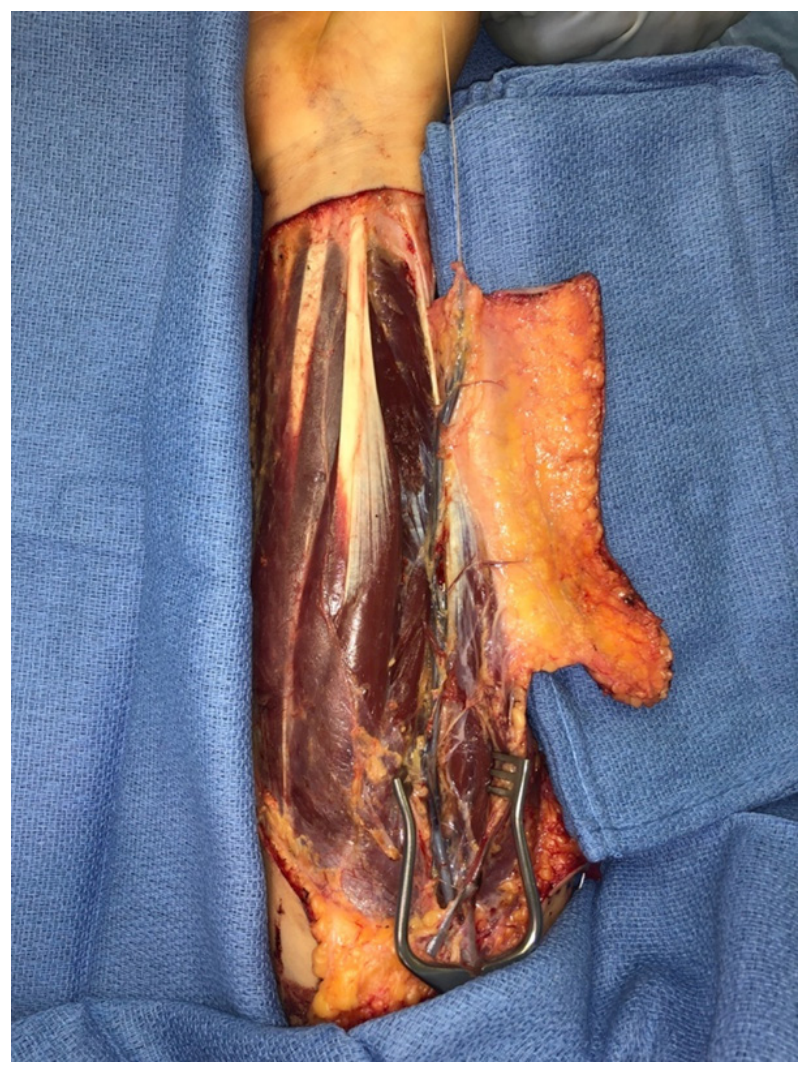

Figure 4. Elevation of radial forearm free flap with proximal radial artery perforator

smokers. We strongly advise that patients have a negative cotinine test three months prior to phalloplasty surgery. Although typical recommendations are to stop smoking 4-6 weeks prior to an elective surgery from a physiologic standpoint, smoking cessation comes with marked psychological stress and as such, it is advisable to deal with this significant life stressor prior to adding the mental and physical stress of complex surgery. Diabetes should be well controlled, with a Hb1Ac within normal limits. While obesity itself is not known to be a risk factor for PFL, it can make the microvascular aspect of the surgery difficult or impossible. We recommend that ideally, patients have a BMI $<30$, although we evaluate any patient with a $\mathrm{BMI}<35$ as fat distribution is a greater determining factor than BMI alone. In the studies that did report on BMI, none assessed whether increased BMI was a risk factor for PFL. It is important for future research to include all relevant co-morbidities and patient demographics. It would be impactful if this information was included both for the total number of patients as well as for the subgroups suffering complications. This would allow future meta-analyses to be performed in order to perform subgroup analysis and identify risk factors for PFL, as well as other complications. It is important to identify modifiable pre-operative risk factors. As previously stated, complication rates are extremely high following phalloplasty surgery. Although these surgeries are essential to this patient population, there is time in the pre-operative assessment period to introduce lifestyle modifications and optimize the medical management of co-morbidities to optimize patient condition, and in doing so, hopefully minimize complication rates. The first step in this process is to identify the risk factors that appear to correlate with specific complications.

\section{A2 flap dimensions}

The dimensions of the RFFF and ALT flap used for phalloplasty are significantly larger than those of a standard RFFF or ALT. This is especially true when performing a tube within tube (TWT) phalloplasty. The ideal TWT flap dimensions are at least $16 \mathrm{~cm}$ in width and $16 \mathrm{~cm}$ or more in length. These dimensions are 
far beyond most traditionally-described ALT and radial forearm flaps, pushing the limits of the perforators and their associated perfasomes to perfuse the flap via linking vessels. De Wolf et al. ${ }^{[17]}$ recognized this issue and utilized intraoperative indocyanine green angiography to identify RFFF that that would benefit from supercharging and did so by using the posterior interosseous artery. In these cases, the anastomosis is performed to the palmar branch of the radial artery distally or in an end-to-side fashion proximally. In their study of 29 patients, 15 had supercharging of the radial forearm flap as described above, resulting in no cases of PFL.

In cases utilizing the ALT flap, dimensions are often even wider than those of the radial forearm. This is because the ALT flap is significantly thicker with a greater proportion of adipose tissue when compared to the radial forearm. This additional bulk often requires a wider skin paddle in order to be able to tube the flap around the thicker adipose tissues. Care has to be taken to include as many perforators as possible in order to support this increased flap volume (see section C1 ALT - absence of or failure to include distal perforators). Intuitively, it makes sense that if an ALT or RFFF is only used for shaft-only and composite flaps, the PFL rate would be lower due to smaller dimensions. The current literature shows a trend in that direction except for the shaft only ALT, which had a markedly higher rate of PFL [Table 3]. However, this is based on a single study that may have been an outlier. Future studies should include flap dimensions to account for lengths that may vary between cohorts and the number of perforators included.

\section{A3 technical errors}

Obviously, any technical errors diminishing perfusion increase the risk for partial or total flap loss. For RFFF, a sub-fascial dissection between the flexor carpi radialis and past the superficial radial nerve can assist in protecting the radial artery and its associated perforators. During ALT harvest, care has to be taken to include all possible perforators, including those with a complex intramusuclar course due to the large dimension of the flap taken and its associated volume of tissue. The time spent meticulously dissecting out all viable perforators will be well-spent by decreasing the risk of areas of PFL as much as possible. Failure to convert to a free flap in case of a short pedicle or failure to create a wide enough tunnel are other technical aspects to consider.

\section{B1 RFFFP - proximal perforator inclusion}

In a traditional antegrade radial forearm flap, only the distal perforators are captured. In the RFFFP, it is important to also capture the proximal perforators ${ }^{[7]}$ [Figure 4]. There is usually one distinct large perforator entering the flap that may exit the radial artery proximal to the proximal flap edge. If unaware of its location and importance, it is easily divided. In our practice, we have realized that this perforator may even take a brief intramuscular or intratendinous course and care should be taken to identify this perforator and dissect it out. Some authors advocate keeping a dermal bridge between the shaft and the proximal urethral extension to further improve blood supply to this watershed region. In the literature, the most commonly reported region of PFL was the distal radial border of the flap; this correlates with our own experience ${ }^{[16]}$. The vascular territory supplied by perforators of the posterior interosseous artery correlates anatomically to the dorsal and radial aspect of the radial forearm flap. Therefore, the previously mentioned strategy of supercharging with use of the posterior interosseous artery may be an ideal strategy to avoid PFL in this location ${ }^{[16]}$.

\section{B2 RFFFP - location}

None of the review articles comment on the exact placement of the RFFFP design on the forearm in regards to the location of the radial artery. Most articles that discuss technique do, however, comment on the benefit of placing the urethra over the ulnar side of the forearm due to the sparse amount of hair follicles in this region. Although a valuable point regarding hair follicle density, this is not always the best strategy in regards to optimizing flap perfusion. In the case of a small forearm circumference, this may 
lead to placement of the flap that is relatively too radial with eccentric location of the radial artery. This can increase the risk of local areas of ischemia and flap loss. In our practice, in patients with a forearm circumference of less than $15.5 \mathrm{~cm}$ we place the flap further ulnar beyond the least hair-bearing area to allow the radial artery to be more centralized. We have no data to support this claim, but it makes logical sense to have the perforators closer to the edge that is most affected by PFL (dorsoradial) and hopefully capture enough linking vessels to allow for perfusion of the entire flap.

\section{C1 ALT - absence of or failure to include distal perforators}

When it comes to ALT phalloplasty, the tissue of the distal aspect of the thigh has two very important benefits compared to harvesting the ALT from the mid-thigh: (1) thinner and more pliable tissue; and (2) a longer vascular pedicle allowing for a comfortable transfer to the genital region. The obvious downside however, is that more distal placement of the flap design often does not allow capture of the proximal and often more sizeable perforators. Additionally, distal perforators are more often intramuscular and necessitate a more difficult intramuscular dissection. Pre-operative CT angiography (CTA) can assist decision-making by identifying the laterality that is most likely to have numerous perforators in the desired flap location and have the highest take off of the lateral femoral circumflex artery, allowing for greater pedicle length for flap transposition ${ }^{[31]}$. In addition to CTA, we routinely perform hand-held Doppler exams in our clinic to document the location of audible perforators. Even in ideal circumstances, it is typical that the most sizeable perforators will often enter the proximal third of the flap. A strategy discussed at oral presentations by the Buncke clinic group (San Francisco) is to include a de-epithelized proximal dermal extension to capture perforators located proximally to the flap design.

\section{C2 ALT - excessive intraoperative thinning}

In most circumstances, the ALT is too bulky for TWT phalloplasty. Even in thin patients, the dimensions of the flap often get too bulky. In these cases, intraoperative thinning of the subscarpal fat layer is a useful strategy to allow for a more natural aesthetic result. We favor doing primary shaping rather than secondary liposuction or lipectomy. However, this is not without consequence. As the lateral femoral cutaneous nerve runs along this fascial plane, it jeopardizes innervation to the neophallus and, if done too excessively, can impede the subcutaneous connecting vascular plexus. In our experience, however, judicious thinning allows for decreased tension on the tissues when they are tubed and comes with improved overall flap vascularity by reducing the overall bulk and size of the flap.

\section{C3 - Failure to delay the flap}

Despite meticulous pre-operative medical and physiological optimization and surgical planning, the size of the flap may be just too big for the few available perforators on which the flap can be elevated. Therefore, it is our opinion that a low threshold should be held to delay a flap that appears congested or marginally perfused in the operating room. We have performed this successfully in two cases. Of course, we cannot know if the flaps would have been adequately perfused if transferred and shaped primarily as originally planned. However, especially with a TWT flap, it makes logical sense that a flap that is already mildly congested will not endure additional strain and insult. In these two cases, we took the patient back on postoperative days 5 and 7, respectively. They both did well and the flaps appeared less congested at the time of take back. There is little risk to flap delay and it may avoid disastrous complications for the patient. A phalloplasty is not like a standard flap in that the shaping is much more aggressive. The flap is being tubed, often twice on itself. Therefore, the vascular inflow and outflow must be robust. If relying only on random pattern perfusion through choke vessels outside of the perfasomes of the flap, the tubing of the flap will almost certainly lead to vascular issues. Therefore, delay should be considered if absolute confidence does not exist in regards to flap vascularity. The overall quality of a reconstruction should never be compromised in an effort to cut down on surgical stages; if necessary, delay. 


\section{Location and extent of partial flap loss}

The location of PFL will dictate both its management and morbidity. Distal radial edge necrosis of a RFFFP can be easily managed with excision and skin grafting. This differs from necrosis in a more critical area such as the proximal urethral extension, which may lead to fistula and stricture formation and the need for multiple reconstructive procedures. In the literature, there was a glaring deficiency in clearly outlining both the anatomic location as well as the extent of PFL. Only 4 of 17 studies reported on the anatomic location of PFL - all mentioned the tip or shaft (distal tissue) and none commented on the loss of tissue in the region of the proximal urethra. This matches our own experience and may be an indication that the proximal urethra may have better blood supply than is often feared [Figure 5]. A discussion on the anatomic location of PFL when referring to the ALT flap is complicated by the confounding factor that surgeons often place the urethra on different sides of the ALT flap design. For example, we like to place the urethra on the lateral aspect of the thigh, while other centers place it medially ${ }^{[32]}$. We believe the vascularity to be better laterally since the angle of the entry point of the perforators point laterally and inferiorly.

Most studies that reported on the timing of glansplasty have delayed it for the ALT cohort as the perforators enter the flap more proximally then they do in a RFFF. The studies with the highest amount of PFL unfortunately did not include this detail. Even if all studies included the timing of glansplasty, we would also need to know if this was done in a sub- or intra-dermal layer as this may affect the outcome.

\section{Management of partial flap loss}

Despite our best efforts, PFL will likely always be a possibility in the field of phalloplasty due to the large flap dimensions necessary for construction of the neophallus. The management of PFL will very much depend on both the anatomic location and extent of tissue loss. When PFL occurs in the urethra, there can be difficulty identifying its occurrence in a timely fashion since this part of the reconstruction is buried either within the shaft or, in the case of single-stage phalloplasty, in the prepubic region. This may delay diagnosis and only become apparent in the setting of an infection or stricture.

\section{Urethral loss}

The management of urethral loss depends on the extent of involvement. Smaller segments can be managed by excision and re-anastomosis, or a two-stage urethroplasty using buccal mucosa grafts. Larger segments of urethral loss may necessitate additional tissue transfer, which adds to the donor site burden and potentially increases the overall complication profile. At our institution, we perform a staged approach to phalloplasty as developed by the St. Peter Andrology Institute. In this strategy, the urethra is marsupialized adjacent to the clitoral shaft at the first stage and as such, is amenable to visible inspection in the postoperative period [Figure 6]. At the second stage, the urethra is connected to the native urethra using vulvar tissue. To allow for this, the ipsilateral labia minorum is resected during the first surgery. If done in this fashion, any ischemia can be identified promptly and the urethra can be shortened and marsupialized at a higher level if necessary [Figure 7].

\section{Shaft loss}

The management strategy regarding the loss of shaft tissue also depends on the extent of involvement. Larger areas may require an additional free flap. In the case of full thickness tissue loss, we favor placement of Integra (Integra, Princeton NJ) followed by the application of a full thickness skin graft. Skin grafting alone may suffice in the setting of partial thickness tissue loss [Figure 5].

\section{CONCLUSION}

The current literature is inconsistent with reports on PFL, and details regarding the anatomic location, extent, and management of PFL are often lacking. This is further complicated by the wide variety of donor sites used for phalloplasty, such that interpretation of the literature becomes even more difficult. In this 

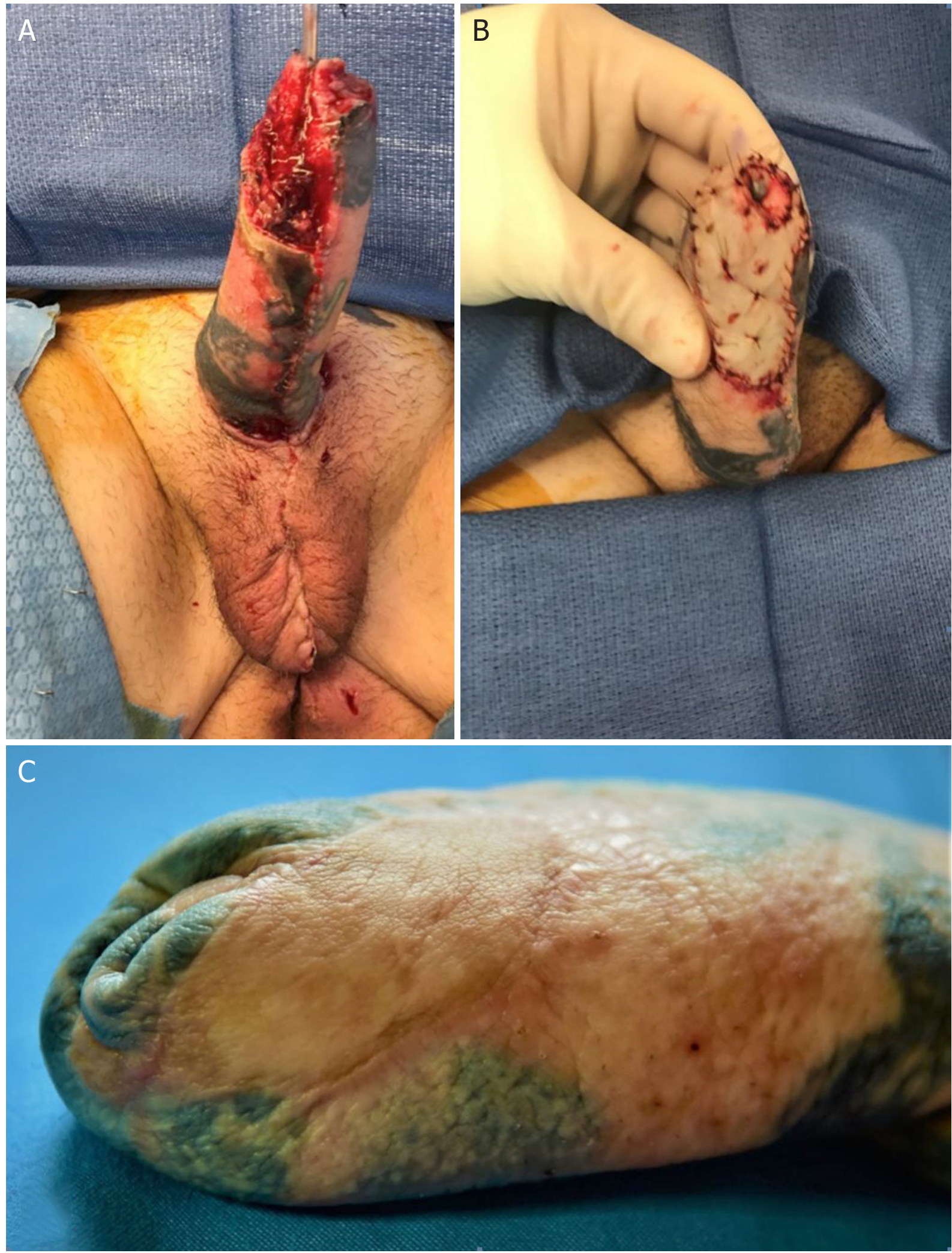

Figure 5. An example of partial flap loss in radial forearm free flap phalloplasty at the distal radial aspect of the flap. A: debridement of the flap and placement of Integra (Integra, Princeton, NJ); B: status post full thickness skin graft (FTSG); C: healed appearance following FTSG $^{[34,35]}$ 


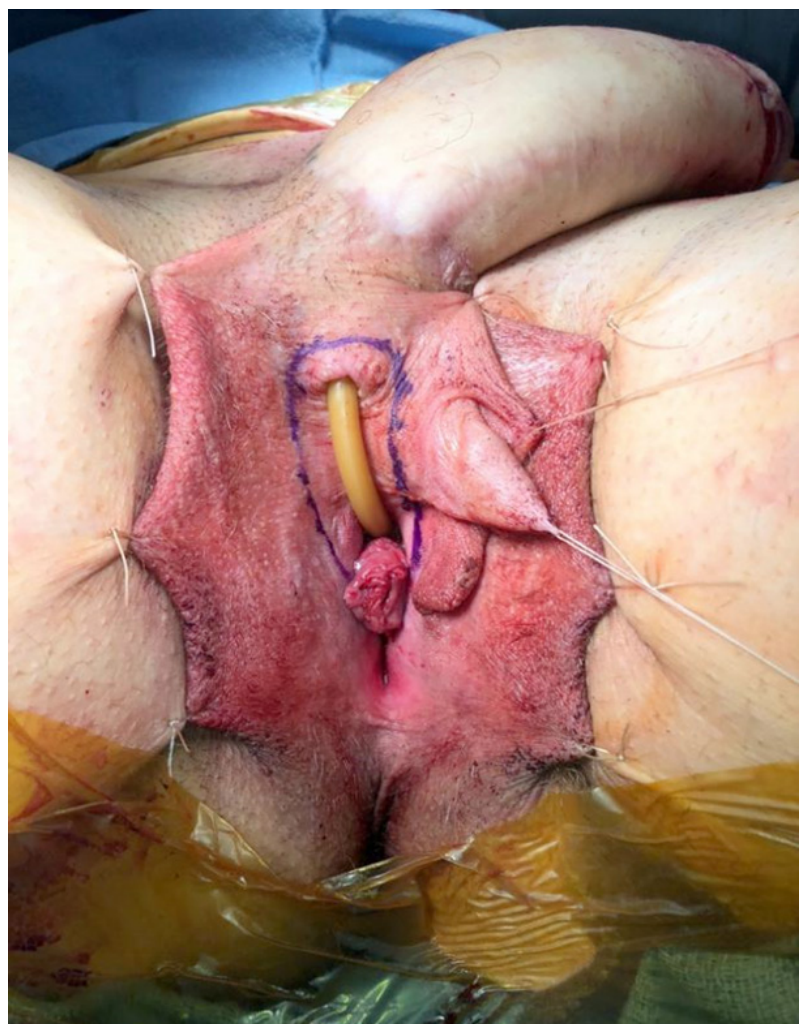

Figure 6. Appearance at the second stage of a Big Ben style two-stage phalloplasty. Note the marsupialized pars pendulans urethra ${ }^{[33,34]}$
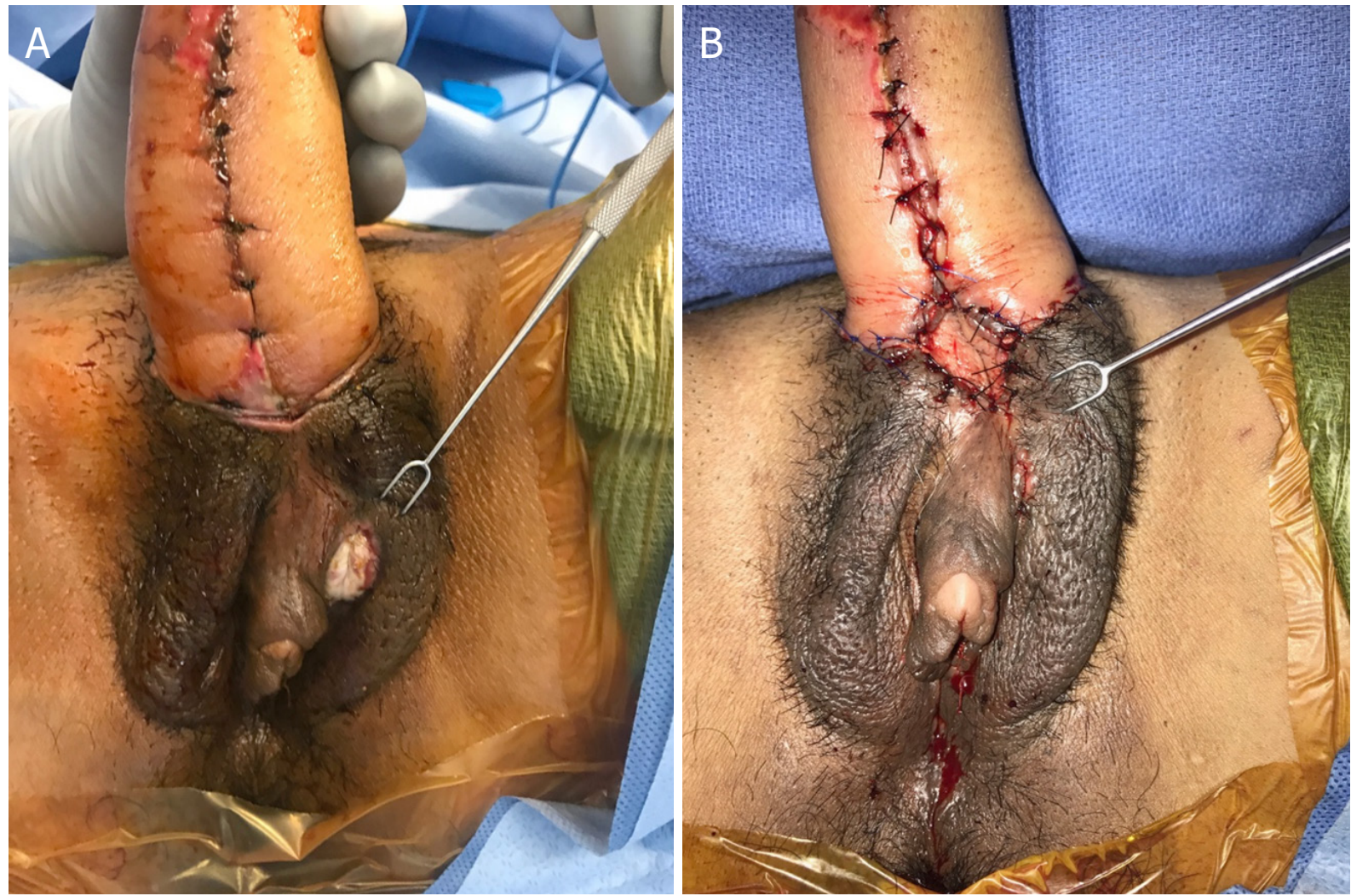

Figure 7. Example of partial flap loss of the proximal urethral extension in an anterolateral thigh flap phalloplasty. After stage two, the patient developed a fistula that was amenable to outpatient repair; voiding now and has received a semi-malleable implant. A: visible ischemic urethral flap; B: status post marsupialization at a more superior location ${ }^{[35]}$ 
systematic review, we focused on the RFFF and ALT flaps used for shaft creation. There seems to be a higher rate of PFL in the ALT cohort than the RFFF cohort (7.1\% vs. $4.5 \%)$. While the rate of total flap loss is historically higher in free flaps compared to pedicled flaps, PFL is not dictated by the type of flap, but rather the dimensions and volume of tissue harvested on a finite number of perforators. Thus, while total flap loss is attributed largely to technical failure, PFL may be considered more of a failure of design. Moreover, the nature of free flaps allows more flexibility regarding vessel lie and pedicle inset, while pedicled flaps may be more constrained three-dimensionally. When discussing PFL, future studies should report on the above variables and strive to include all pertinent patient demographics, flap characteristics, and outcomes. It is only through the identification of risk factors for PFL that appropriate strategies to mitigate and treat this complication will become apparent.

\section{DECLARATIONS}

\section{Authors' contributions}

Manuscript preparation, literature search, data review, creation of tables: Cylinder I

Data review, creation of tables: Heston A

Data review, creation of tables: Jedrzejewski B

Manuscript preparation, data review: Sikora Z

Manuscript preparation, data review: Peters B

Manuscript preparation, data review: Berli JU

\section{Availability of data and materials}

The authors confirm that the data supporting the findings of this study are available within the article.

\section{Financial support and sponsorship}

None.

\section{Conflicts of interest}

All authors declared that there are no conflicts of interest.

\section{Ethical approval and consent to participate}

Not applicable.

\section{Consent for publication}

Not applicable.

\section{Copyright}

(c)The Author(s) 2020.

\section{REFERENCES}

1. Gillies H. Congenital absence of the penis: with embryological considerations. Br J Plast Surg 1948;1:8-28.

2. Kim S, Dennis M, Holland J, Terrell M, Loukas M, et al. The anatomy of abdominal flap phalloplasty for transgender surgery. Clin Anat 2018;31:181-6.

3. Puckett CL, Reinisch JF, Montie JE. Free flap phalloplasty. J Urol 1982;128:294-7.

4. Chang TS, Hwang WY. Forearm flap in one-stage reconstruction of the penis. Plast Reconstr Surg 1984;74:251-8.

5. Hage JJ, De Graaf FH. Addressing the ideal requirements by free flap phalloplasty: some reflections on refinements of technique. Microsurgery 1993;14:592-8.

6. Djordjevic ML, Bencic M, Kojovic V, Stojanovic B, Bizic M, et al. Musculocutaneous latissimus dorsi flap for phalloplasty in female to male gender affirmation surgery. World J Urol 2019;37:631-7.

7. Gottlieb LJ. Radial forearm. Clin Plast Surg 2018;45:391-8.

8. Xu KY, Watt AJ. The pedicled anterolateral thigh phalloplasty. Clin Plast Surg 2018;45:399-406.

9. Zaheer U, Granger A, Ortiz A, Terrell M, Loukas M, et al. The anatomy of free fibula osteoseptocutaneous flap in neophalloplasty in 
transgender surgery. Clin Anat 2018;31:169-74.

10. Nikolavsky D, Hughes M, Zhao LC. Urologic complications after phalloplasty or metoidioplasty. Clin Plast Surg 2018;45:425-35.

11. Nikolavsky D, Yamaguchi Y, Levine JP, Zhao LC. Urologic sequelae following phalloplasty in transgendered patients. Urol Clin North Am 2017;44:113-25.

12. Morrison SD, Shakir A, Vyas KS, Kirby J, Crane CN, et al. Phalloplasty: a review of techniques and outcomes. Plast Reconstr Surg 2016;138:594-615.

13. Al-Tamimi M, Pigot GL, van der Sluis WB, van de Grift TC, van Moorselaar RJA, et al. The surgical techniques and outcomes of secondary phalloplasty after metoidioplasty in transgender men: an international, multi-center case series. J Sex Med 2019;16:1849-59.

14. Ascha M, Massie JP, Morrison SD, Crane CN, Chen ML. Outcomes of single stage phalloplasty by pedicled anterolateral thigh flap versus radial forearm free flap in gender confirming surgery. J Urol 2018;199:206-14.

15. Baumeister S, Sohn M, Domke C, Exner K. Phalloplasty in female-to-male transsexuals: experience from 259 cases. Handchir Mikrochir Plast Chir 2011;43:215-21.

16. D'Arpa S, Claes K, Lumen N, Oieni S, Hoebeke P, et al. Urethral reconstruction in anterolateral thigh flap phalloplasty: a 93-case experience. Plast Reconstr Surg 2019;143:382-92e.

17. De Wolf E, Claes K, Sommeling CE, Opsomer D, Cherubino M, et al. Free bipedicled radial forearm and posterior interosseous artery perforator flap phalloplasty. J Sex Med 2019;16:1111-7.

18. Fang RH, Kao YS, Ma S, Lin JT. Phalloplasty in female-to-male transsexuals using free radial osteocutaneous flap: a series of 22 cases. Br J Plast Surg 1999;52:217-22.

19. Fang RH, Lin JT, Ma S. Phalloplasty for female transsexuals with sensate free forearm flap. Microsurgery 1994;15:349-52.

20. Felici N, Felici A. A new phalloplasty technique: the free anterolateral thigh flap phalloplasty. J Plast Reconstr Aesthet Surg 2006;59:153-7.

21. Hage JJ, Bouman FG, de Graaf FH, Bloem JJ. Construction of the neophallus in female-to-male transsexuals: the Amsterdam experience. J Urol 1993;149:1463-8.

22. Kim SK, Lee KC, Kwon YS, Cha BH. Phalloplasty using radial forearm osteocutaneous free flaps in female-to-male transsexuals. J Plast Reconstr Aesthet Surg 2009;62:309-17.

23. Küntscher AM, Kilian M, Bull S, Kuntscher MV. The radial forearm flap with a prelaminated urethra: analysis of complications based on the Clavien-Dindo classification. Handchir Mikrochir Plast Chir 2019.

24. Leriche A, Timsit MO, Morel-Journel N, Bouillot A, Dembele D, et al. Long-term outcome of forearm flee-flap phalloplasty in the treatment of transsexualism. BJU Int 2008;101:1297-300.

25. Matti BA, Matthews RN, Davies DM. Phalloplasty using the free radial forearm flap. Br J Plast Surg 1988;41:160-4.

26. Namba Y, Watanabe T, Kimata Y. Flap combination phalloplasty in female-to-male transsexuals. J Sex Med 2019;16:934-41.

27. Song C, Wong M, Wong CH, Ong YS. Modifications of the radial forearm flap phalloplasty for female-to-male gender reassignment. J Reconstr Microsurg 2011;27:115-20.

28. van der Sluis WB, Smit JM, Pigot GLS, Buncamper ME, Winters HAH, et al. Double flap phalloplasty in transgender men: surgical technique and outcome of pedicled anterolateral thigh flap phalloplasty combined with radial forearm free flap urethral reconstruction. Microsurgery 2017;37:917-23.

29. Wirthmann AE, Majenka P, Kaufmann MC, Wellenbrock SV, Kasper L, et al. Phalloplasty in female-to-male transsexuals by gottlieb and levine's free radial forearm flap technique-a long-term single-center experience over more than two decades. J Reconstr Microsurg 2018;34:235-41.

30. Hwang K, Son JS, Ryu WK. Smoking and flap survival. Plast surg (Oakv) 2018;26:280-5.

31. Annen AW, Heston AL, Dugi DD III, Dy GW, Bluebond-Langner R, et al. Masculinizing genital surgery: an imaging primer for the radiologist. AJR Am J Roentgenol 2020;214:W27-36.

32. Chen ML, Safa B. Single-stage phalloplasty. Urol Clin North Am 2019;46:567-80.

33. Danker S, Esmonde N, Berli JU. "Staging” in Phalloplasty. Urol Clin North Am 2019;46:581-90.

34. Heston AL, Esmonde NO, Dugi DD, Berli JU. Phalloplasty: techniques and outcomes. Transl Androl Urol 2019;8:254-65.

35. Esmonde N, Bluebond-Langner R, Berli JU. Phalloplasty flap-related complication. Clin Plast Surg 2018;45:415-24. 